Physics

Physics Research Publications

\title{
Pairing Symmetry in a Two-Orbital Exchange Coupling Model of Oxypnictides
}
K. J. Seo
B. A. Bernevig

J. P. Hu 


\title{
Pairing Symmetry in a Two-Orbital Exchange Coupling Model of Oxypnictides
}

\author{
Kangjun Seo, ${ }^{1}$ B. Andrei Bernevig, ${ }^{2}$ and Jiangping $\mathrm{Hu}^{1}$ \\ ${ }^{1}$ Department of Physics, Purdue University, West Lafayette, Indiana 47907, USA \\ ${ }^{2}$ Princeton Center for Theoretical Science, Princeton University, Princeton, New Jersey 08544, USA
}

(Received 3 June 2008; published 14 November 2008)

\begin{abstract}
We study the pairing symmetry of a two-orbital $J_{1}-J_{2}$ model for FeAs layers in oxypnictides. We show that the mixture of an intraorbital unconventional $s_{x^{2} y^{2}} \sim \cos \left(k_{x}\right) \cos \left(k_{y}\right)$ pairing symmetry, which changes sign between the electron and hole Fermi surfaces, and a very small $d_{x^{2}-y^{2}} \sim \cos \left(k_{x}\right)-$ $\cos \left(k_{y}\right)$ component is favored in a large part of the $J_{1}-J_{2}$ phase diagram. A pure $s_{x^{2} y^{2}}$ pairing state is favored for $J_{2}>J_{1}$. The signs of the $d_{x^{2}-y^{2}}$ order parameters in the two different orbitals are opposite. While a small $d_{x y} \sim \sin \left(k_{x}\right) \sin \left(k_{y}\right)$ interorbital pairing coexists in the above phases, the intraorbital $d_{x y}$ pairing is not favored even for large $J_{2}$.
\end{abstract}

PACS numbers: 71.27.+a, 71.10.- w, 74.25.Ha

High temperature superconductivity (at $56 \mathrm{~K}$ ) has been recently reported in the rare-earth electron- and hole-doped oxypnictide compounds [1-6]. Preliminary evidence [7-9] suggests that the superconducting state in the electrondoped oxypnictides, like that in the cuprates, has gapless nodal quasiparticles and an unconventional pairing symmetry. A number of theoretical studies have predicted or conjectured different possible pairing symmetries, anywhere from $p$ wave to a $\pi$-shifted $s$ wave [10].

The electronic properties of oxypnictides differ from those in cuprates in several important ways. Most importantly, the undoped oxypnictides are metallic but their resistivity is strikingly high. They can hence be interpreted both as a bad metal or as a poor insulator, leaving open the question of whether a weak or strong coupling fixed point governs their physics. From the band structure point of view, barring the existence of unphysically strong crystal fields, it seems likely that all $3 d$ orbitals of the Fe atoms are involved in the low energy electronic properties. Numerical results based on first principle calculations show the presence of small Fermi surfaces [11]. In the unfolded Brillouin zone consisting of one Fe per unit cell, electron and hole pockets exist around the $M$ and $\Gamma(\pi, \pi)$ points, respectively. The magnetic properties of the oxypnictides are also different from those of the cuprates. Neutron experiments have shown that the magnetic structure in undoped oxypnictides is not a simple antiferromagnetic order [12] but rather a stripe spin-density wave with onset temperature of about $150 \mathrm{~K}$.

The metallic behavior and the existence of Fermi pockets have led to proposals about the superconducting mechanism which originate in the weak coupling, itinerant limit $[13,14]$. However, numerical and analytic research suggests that the antiferromagnetic exchange coupling between Fe sites is strong [15-17]. Because of As-mediated hopping, antiferromagnetic exchange exists not only between the nearest-neighbor (NN) Fe sites but also between next-nearest-neighbor (NNN) sites. The NNN coupling strength $J_{2}$ is comparable to the NN coupling strength $J_{1}$.
The $J_{1}-J_{2}$ model provides for half-filled magnetic physics consistent with experimental neutron data [12]. A nematic magnetic phase transition has been predicted in this model $[18,19]$, consistent with the experimental observation of a structural transition preceding the spin-density wave formation. Therefore, the magnetic structure of the undoped oxypnictides is consistent with strong-correlation physics.

In the present Letter we obtain the superconducting mean-field phase diagram of a $t-J_{1}-J_{2}$ model with the correct Fermi surface for the oxypnictide compounds. We consider only time-reversal symmetric superconducting order parameters, and predict that two kinds of intraorbital pairing order parameters, an extended $s$ wave of the unconventional form $s_{x^{2} y^{2}} \sim \cos k_{x} \cos k_{y}$ or a $d_{x^{2}-y^{2}} \sim$ $\cos k_{x}-\cos k_{y}$ wave order parameter are the only possibilities in the Mott limit. For the experimentally relevant situation $J_{2}>J_{1}$, we predict an $s_{x^{2} y^{2}} \sim \cos k_{x} \cos k_{y}$ order parameter which changes sign between the electron and hole Fermi surfaces. A mixture of the intraorbital unconventional $s_{x^{2} y^{2}}$ pairing symmetry and a small component of $d_{x^{2}-y^{2}}$ pairing symmetry is favored for $J_{1} \approx J_{2}$ (Fig. 1). While a small $d_{x y} \sim \sin \left(k_{x}\right) \sin \left(k_{y}\right)$ interorbital pairing order coexists in the above phase, the intraorbital $d_{x y}$ pairing symmetry is not favored even for large values of $J_{2}$ in contradiction with the predictions of several papers $[17,19,20]$ that rest on an analogy with the physics in cuprates. While $d_{x y}$ pairing would indeed be favored for $J_{2} \gg J_{1}$ in the case of a large, single band, Fermi surface (as in the cuprates) [21], if the oxypnictide local-density approximation Fermi surface picture is correct, we can argue, on general grounds, that $d_{x y}$ pairing cannot compete with the extended $s$ wave we propose even if $J_{2}$ is very large. If we consider a single band and treat a NNN $J_{2}$ in mean-field decoupling, the superconducting transition temperature $T_{c}$ is self-consistently determined by an Eliashberg equation $2 T_{c}=J_{2} \sum_{k}[f(k)]^{2} g\left(x\left(k, T_{c}\right)\right)$ where $f(k)$ is the pairing symmetry factor and $g(x)=\frac{\tanh (x)}{x}$ [with $\left.x\left(k, T_{c}\right)=\frac{\epsilon(k)-\mu}{2 T_{c}}\right]$ positive and peaked at the different 


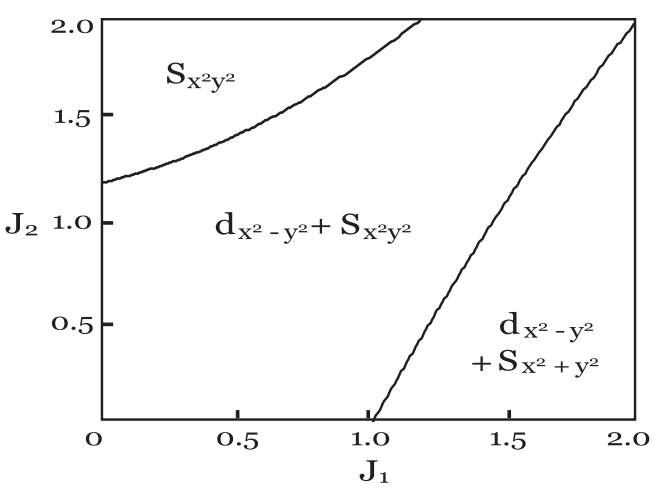

FIG. 1. The superconducting phase diagram in the $J_{1}-J_{2}$ plane at $18 \%$ electron doping obtained by solving the self-consistent Eqs. (3).

Fermi surfaces. Hence $T_{c}$ follows the maximum value of the pairing symmetry factor $|f(k)|$ close to the Fermi surfaces. The $d_{x y}$ pairing symmetry factor, $\sin k_{x} \sin k_{y}$, is always small at the electron $[(0, \pi),(\pi, 0)]$ and hole pockets $[(0,0),(\pi, \pi)$,$] in the unfolded Brillouin zone of$ oxypnictides. Hence $d_{x y}$ pairing symmetry is not favored even for large $J_{2}$.

The model.-We focus on a two-orbital per site model of the oxypnictides, with hybridization between the $d_{x z}$ and $d_{y z}$ orbitals. Although this description is only valid in the case of unphysically large crystal field splitting, we particularize to this model for analytic simplicity. We adopt the band structure proposed in Ref. [14], $H_{0}=$ $\sum_{k \sigma} \psi_{k \sigma}^{\dagger} T(k) \psi_{k \sigma}$,

$$
T(k)=\left(\begin{array}{cc}
\epsilon_{x}(k)-\mu & \boldsymbol{\epsilon}_{x y}(k) \\
\epsilon_{x y}(k) & \boldsymbol{\epsilon}_{y}(k)-\mu
\end{array}\right)
$$

where $\psi_{k, \sigma}^{\dagger}=\left(c_{d_{x z}, k, \sigma}^{\dagger}, c_{d_{y z}, k, \sigma}^{\dagger}\right)$ is the creation operator for spin $\sigma$ electrons in the two orbitals and

$$
\begin{aligned}
& \boldsymbol{\epsilon}_{x}\left(k_{x}, k_{y}\right)=-2 t_{1} \cos k_{x}-2 t_{2} \cos k_{y}-4 t_{3} \cos k_{x} \cos k_{y}, \\
& \boldsymbol{\epsilon}_{y}\left(k_{x}, k_{y}\right)=\boldsymbol{\epsilon}_{x}\left(k_{y}, k_{x}\right), \quad \boldsymbol{\epsilon}_{x y}(k)=-4 t_{4} \sin k_{x} \sin k_{y} .
\end{aligned}
$$

The hoppings have the magnitudes: $t_{1}=-1.0, t_{2}=1.3$, $t_{3}=-0.85, t_{4}=-0.85$ (the energy unit of parameters in this Letter will be set by $\left.\left|t_{1}\right|\right)$. The half-filled, two electrons per site configuration requires $\mu=1.54$. The interaction Hamiltonian contains three terms: the first two are antiferromagnetic $\mathrm{NN}$ and $\mathrm{NNN}$ couplings between the spin of identical and opposite orbitals:

$$
H_{i}=\sum_{r \alpha \beta} J_{i \alpha \beta}\left[\vec{S}(r, \alpha) \cdot \vec{S}\left(r+\delta_{i}, \beta\right)-n(r, \alpha) n\left(r+\delta_{i}, \beta\right)\right]
$$

where $\vec{S}(r, \alpha)=c_{\alpha, r, \gamma}^{\dagger} \vec{\sigma}_{\gamma \gamma^{\prime}} c_{\alpha, r, \gamma^{\prime}}$ is the local spin operator, $n(r, \alpha)$ is the local density operator, $\alpha, \beta$ are orbital index, $i=1,2, \delta_{1}$ is the nearest neighbor and $\delta_{2}$ is the nextnearest neighbor. The third is a Hund's rule coupling of the spins on different orbitals, on the same site: $H_{3}=$ $-\sum_{r \alpha} J_{H} \vec{S}(r, \alpha) \vec{S}(r, \bar{\alpha})$, where $\bar{\alpha}$ is the orbital complementary to $\alpha$. The antiferromagnetic $J_{1}$ and $J_{2}$ (both positive) are usually obtained from numerical calculations involving overlap with Fe-As-Fe orbitals. Our mean-field solutions should be interpreted in the same spirit as the superconducting solutions of the original $t-J$ model: at some value of the doping, the true undoped spin-density wave groundstate disappears and gives way to a superconducting state [22].

Keeping all of the above terms becomes analytically intractable. We proceed with a two-step process: we first mean-field decouple the interaction Hamiltonian assuming that exchange takes place only between spins on the same orbitals. While physically incomplete, this model is analytically tractable, and exposes the uncompetitiveness of $d_{x y}$ order. We then numerically solve the full model. The interaction term reads $\sum_{k, k^{\prime}} V_{k, k^{\prime}} c_{\alpha, k, \uparrow}^{\dagger} c_{\alpha,-k, \downarrow}^{\dagger} c_{\alpha,-k^{\prime}, \downarrow} c_{\alpha, k^{\prime}, \uparrow}$ with $\quad V_{k, k^{\prime}}=-\frac{2 J_{1}}{N} \sum_{ \pm}\left(\cos k_{x} \pm \cos k_{y}\right)\left(\cos k_{x}^{\prime} \pm \cos k_{y}^{\prime}\right)-$ $\frac{8 J_{2}}{N}\left(\cos k_{x} \cos k_{y} \cos k_{x}^{\prime} \cos k_{y}^{\prime}+\sin k_{x} \sin k_{y} \sin k_{x}^{\prime} \sin k_{y}^{\prime}\right)$ with obvious pairing amplitudes in four channels $x^{2} \pm y^{2}, x y$, and $\quad x^{2} y^{2}, \quad \Delta_{\alpha}(k)=\Delta_{x^{2}+y^{2}, \alpha}(k)+\Delta_{x^{2}-y^{2}, \alpha}(k)+$ $\Delta_{x^{2} y^{2}, \alpha}(k)+\Delta_{x y, \alpha}(k)$, and

$$
\begin{aligned}
\frac{\Delta_{x^{2} \pm y^{2}, \alpha}(k)}{\cos k_{x} \pm \cos k_{y}} & =-\frac{2 J_{1}}{N} \sum_{k^{\prime}}\left(\cos k_{x}^{\prime} \pm \cos k_{y}^{\prime}\right) d\left(k^{\prime}\right), \\
\frac{\Delta_{a, \alpha}(k)}{f_{a}(k)} & =-\frac{8 J_{2}}{N} \sum_{k^{\prime}}\left[f_{a}\left(k^{\prime}\right)\right] d\left(k^{\prime}\right)
\end{aligned}
$$

where $a=x^{2} y^{2}, x y, f_{a}(k)$ are corresponding symmetry factors and $d\left(k^{\prime}\right)=\left\langle c_{\alpha,-k^{\prime}, \downarrow} c_{\alpha, k^{\prime}, \uparrow}\right\rangle$. We use $\alpha=\{1,2\}$ to represent the orbital index $(x z, y z)$.

We decouple the interaction Hamiltonian with exchange terms only between spins on the same orbitals in meanfield: $H_{m}=\sum_{k} \Psi(k)^{\dagger} A(k) \Psi(k)$ with

$$
A(k)=\left(\begin{array}{cccc}
\epsilon_{x}(k)-\mu & \Delta_{1}(k) & \boldsymbol{\epsilon}_{x y}(k) & 0 \\
\Delta_{1}^{*}(k) & -\epsilon_{x}(k)+\mu & 0 & -\boldsymbol{\epsilon}_{x y}(k) \\
\epsilon_{x y}(k) & 0 & \boldsymbol{\epsilon}_{y}(k)-\mu & \Delta_{2}(k) \\
0 & -\epsilon_{x y}(k) & \Delta_{2}^{*}(k) & -\boldsymbol{\epsilon}_{y}(k)+\mu
\end{array}\right)
$$

with $\Psi(k)=\left(c_{1, k, \uparrow}, c_{1,-k, \downarrow}^{\dagger}, c_{2, k, \uparrow}, c_{2,-k, \downarrow}^{\dagger}\right)$. The particularization to oxypnictides is present in the hopping terms, which couple different orbitals as in Eq. (2). $A(k)$ can be diagonalized by an unitary transformation, $U(k)^{\dagger} A(k) U(k)$, and the Bogoliubov quasiparticle eigenvalues $E_{1}=-E_{2}$ and $E_{3}=-E_{4}$ are given by

$$
E_{m=1,3}(k)=\frac{1}{\sqrt{2}} \sqrt{\left(\tilde{\boldsymbol{\epsilon}}_{x}^{2}+\tilde{\boldsymbol{\epsilon}}_{y}^{2}+2 \epsilon_{x y}^{2}+\Delta_{1}^{2}+\Delta_{2}^{2}\right) \pm \sqrt{\left(\tilde{\boldsymbol{\epsilon}}_{x}^{2}-\tilde{\boldsymbol{\epsilon}}_{y}^{2}+\Delta_{1}^{2}-\Delta_{2}^{2}\right)^{2}+4 \epsilon_{x y}^{2}\left[\left(\tilde{\boldsymbol{\epsilon}}_{x}+\tilde{\boldsymbol{\epsilon}}_{y}\right)^{2}+\left(\Delta_{1}-\Delta_{2}\right)^{2}\right]}}
$$


where $\tilde{\epsilon}_{x, y}=\epsilon_{x, y}-\mu$. The self-consistent gap and density equations are

$$
\begin{aligned}
\Delta_{1,2}(k) & =\sum_{k^{\prime}, m} V_{k, k^{\prime}} U_{2,4 m}^{*}\left(k^{\prime}\right) U_{1,3 m}\left(k^{\prime}\right) F\left[E_{m}\left(k^{\prime}\right)\right], \\
n_{(1,2)} & =2 \sum_{k^{\prime}, m} U_{(1,3) m}^{*}\left(k^{\prime}\right) U_{(1,3) m}\left(k^{\prime}\right) F\left[E_{m}\left(k^{\prime}\right)\right]
\end{aligned}
$$

where $F(E)$ is the Fermi-Dirac distribution function, $F(E)=\frac{1}{1+e^{E / k T}}$. To obtain the transition temperature, we linearize the self-consistent equation for small $\Delta_{1}, \Delta_{2}$. After tedious algebra, we find the self-consistent equations around $T_{c}$ :

$$
\Delta_{2}(k)=\sum_{k^{\prime}} V_{k, k^{\prime}}\left[W_{3}\left(k^{\prime}\right)-W_{1}\left(k^{\prime}\right)\right]
$$

where

$W_{i}=\frac{\left[\left(\epsilon_{x}-\mu\right)^{2}-\tilde{E}_{i}^{2}\right] \Delta_{2}+\epsilon_{x y}^{2} \Delta_{1}}{2\left|\epsilon_{x}+\epsilon_{y}-2 \mu\right| \tilde{E}_{i} \sqrt{4 \epsilon_{x y}^{2}+\left(\epsilon_{x}-\epsilon_{y}\right)^{2}}} \tanh \left(\beta \tilde{E}_{i} / 2\right)$

with $\tilde{E}_{i}=E_{i}\left(\Delta_{1}=\Delta_{2}=0\right)$.

The above equations can be solved numerically, varying the doping $\mu$ and the value of $J_{1}$ and $J_{2}$. In Fig. 1, we plot the phase diagram in the $J_{1}-J_{2}$ plane with $18 \%$ electron doping. The phase on the left upper corner where $J_{2}>$ $J_{2 c} \sim 1.2$ has pure extended $s$-wave pairing symmetry $s_{x^{2} y^{2}}$ phase. The phase on the right side, where $J_{1}>J_{1 c} \sim 1.05$, is a mixture of $s_{x^{2}+y^{2}}$ and a small amount of $d_{x^{2}-y^{2}}$. The remaining large part of phase diagram is described by a phase with mixed $s_{x^{2} y^{2}}$ and small $d_{x^{2}-y^{2}}$ pairing order parameters. In this mixed state, the signs of the $d_{x^{2}-y^{2}}$ order parameters in the two orbitals are opposite. Namely, if $\Delta_{1}=a \cos \left(k_{x}\right) \cos \left(k_{y}\right)+b\left[\cos \left(k_{x}\right)-\cos \left(k_{y}\right)\right], \quad \Delta_{2}=$ $a \cos \left(k_{x}\right) \cos \left(k_{y}\right)-b\left[\cos \left(k_{x}\right)-\cos \left(k_{y}\right)\right]$. We do not find a $d_{x y}$ solution in the entire parameter region. Time-reversal broken states, such as $s+i d$, are not favored.

The above results can be understood analytically. First, we can plot a pairing weight $W_{3}-W_{1}$ as a function of the Brillouin zone momentum $\left(k_{x}, k_{y}\right)$ (Fig. 2) by taking $\Delta_{2}=$ $\Delta_{1}=1$ in Eq. (9). The values of order parameters are determined by the pairing symmetry factor function times this quantity. The dominant contribution is clearly around $\Gamma, M$ and $(\pi, \pi)$. The $d_{x y}$ order, in which the pairing symmetry factor, $\sin k_{x} \sin k_{y}$, is peaked around $( \pm \pi / 2$, $\pm \pi / 2$ ) has small overlap with the pairing weight and is not favored. Second, the mixing strength of two order parameters is determined by multiplying the two symmetry factors $\left(f_{1}, f_{2}\right)$ of two order parameters and the paring weight: $\sum_{k} f_{1}(k) f_{2}(k)\left[W_{3}(k)-W_{1}(k)\right]$. It is easy to check, for a mixture of $s_{x^{2} y^{2}}$ and $d_{x^{2}-y^{2}}$, i.e., $f_{1}=\cos k_{x} \cos k_{y}$, $f_{2}=\left(\cos k_{x}-\cos k_{y}\right)$ that the summation has a large contribution from the Brillouin zone momentum around the electron pocket. The mixture strength of the other two order parameters $\left(s_{x^{2} y^{2}}\right.$ and $\left.s_{x^{2}+y^{2}}\right)$ is very small. This

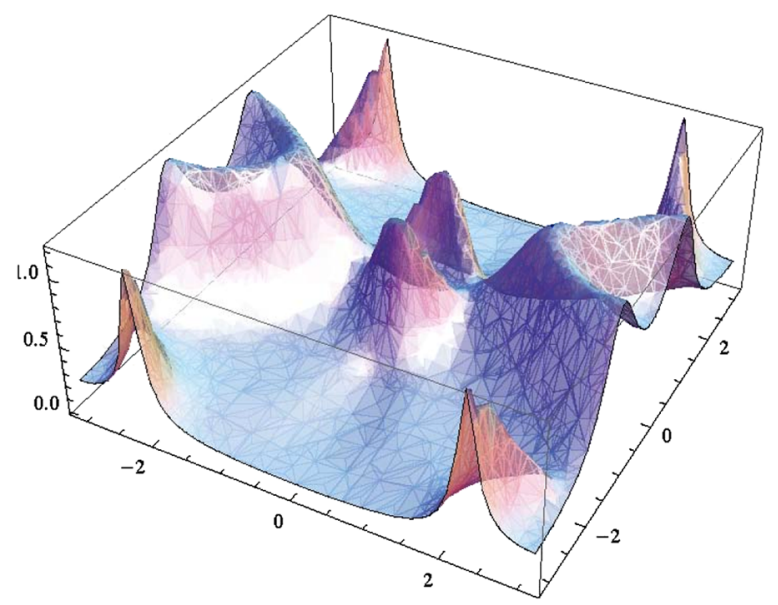

FIG. 2 (color online). $3 \mathrm{D}$ plot of the pairing weight $W_{3}-W_{1}$ as a function of $\left(k_{x}, k_{y}\right)$ (electron doped) by setting $\Delta_{1}=\Delta_{2}=1$ in Eq. (9).

explains why the phase diagram is dominated by the mixture of $s_{x^{2} y^{2}}$ and a small amount of $d_{x^{2}-y^{2}}$. Finally, the difference of the relative sign between the $s_{x^{2} y^{2}}$ and $d_{x^{2}-y^{2}}$ order parameters in the two different orbitals is a result of the fact that exchanging $k_{x}$ to $k_{y}$ maps the $x z$ to the $y z$ orbital.

The part of the phase diagram in Fig. 1 with mixed $s_{x^{2} y^{2}}$ and small $d_{x^{2}-y^{2}}$ pairing becomes larger as the electron doping concentration is reduced: the mixing strength of $s_{x^{2} y^{2}}$ and $d_{x^{2}-y^{2}}$ order parameters is (very slightly) increased due to the enhanced contribution around the $M$ points. In Fig. 3, we plot the transition temperature as a function of electron doping level at the fixed values of $J_{1}=$ 0.25 and $J_{2}=0.5$. On the electron-doped side, $T_{c}$ is reduced by increasing the doping concentration. This is similar to Ref. [23] and it is, of course, around half-filling, an artifact of the mean-field solution. The true ground state at half-filling is a spin-density wave [24] which gives way to a superconductor as the filling is increased [25].

Solutions including orbital-crossing exchange and Hunds coupling.-We now consider the orbital-crossing exchange antiferromagnetic coupling, $J_{1 ; 12}, J_{2 ; 12}$ and Hunds coupling $J_{H}$. In mean field, we can decouple it in the particle-particle channel. The orbital-crossing exchange coupling can be decoupled in four spin-singlet orbital-crossing pairing order parameters, $\Delta^{\prime}(k)=$ $\Delta_{x^{2}+y^{2}}^{\prime}(k)+\Delta_{x^{2}-y^{2}}^{\prime}(k)+\Delta_{x^{2} y^{2}}^{\prime}(k)+\Delta_{x y}^{\prime}(k)$. Hunds coupling can be decoupled to an on-site spin-triplet, orbitalsinglet, order parameter, $\Delta_{H}=\sum_{k}\left\langle c_{1, k, \uparrow} c_{2,-k, \downarrow}-\right.$ $\left.c_{2, k, \uparrow} c_{1,-k, \downarrow}\right\rangle$. The new mean-field Hamiltonian is $H_{m}^{\prime}=$ $\sum_{k} \Psi(k)^{\dagger} B(k) \Psi(k)$ with $B(k)=A(k)+\delta A(k)$

$$
\delta A(k)=\left(\begin{array}{cccc}
0 & 0 & 0 & \Delta^{\prime}+\Delta_{H} \\
0 & 0 & \Delta^{\prime *}-\Delta_{H}^{*} & 0 \\
0 & \Delta^{\prime}-\Delta_{H} & 0 & 0 \\
\Delta^{\prime *}+\Delta_{H}^{*} & 0 & 0 & 0
\end{array}\right) .
$$



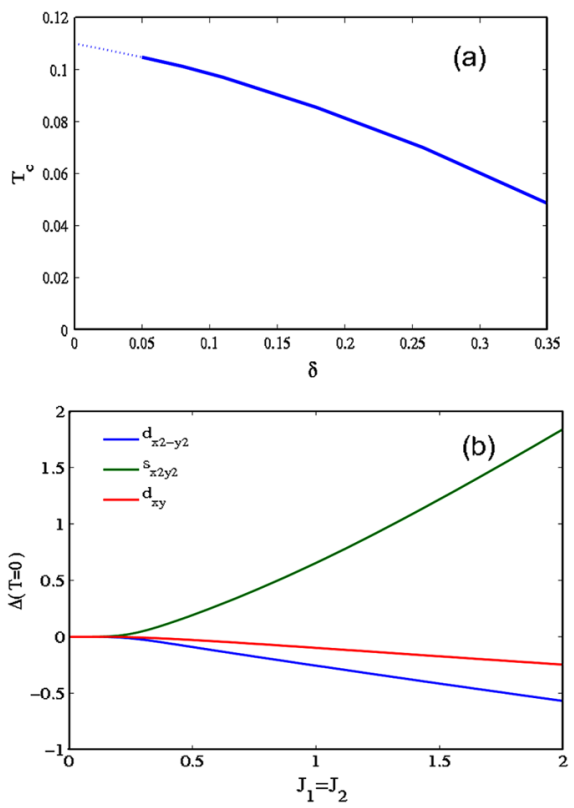

FIG. 3 (color online). (a) The pairing transition temperature as a function of the electron doping concentration at $J_{1}=0.25$ and $J_{2}=0.5$. The dotted line indicates the region where the spindensity wave competing phase takes over from the superconducting phase. (b) The intraorbital, $s_{x^{2} y^{2}}, d_{x^{2}-y^{2}}$ and the interorbital, $d_{x y}$, pairing order parameter, as a function of $J=J_{1}=J_{2}$ when the chemical potential is $\mu=1.8$.

We have an additional self-consistent equation:

$$
\Delta^{\prime}(k)+\Delta_{H}=\sum_{k^{\prime}, m} V_{k, k^{\prime}}^{\prime} U_{4 m}^{\prime *}\left(k^{\prime}\right) U_{1 m}^{\prime}\left(k^{\prime}\right) F\left(E_{m}^{\prime}\left(k^{\prime}\right)\right)
$$

where the interorbital potential $V_{k, k^{\prime}}^{\prime}$ contains NN coupling $-\frac{2 J_{1: 12}}{N} \sum_{ \pm}\left(\cos k_{x} \pm \cos k_{y}\right)\left(\cos k_{x}^{\prime} \pm \cos k_{y}^{\prime}\right)$, a NNN coupling $\quad-\frac{8 J_{2 ; 12}}{N}\left(\cos k_{x} \cos k_{y} \cos k_{x}^{\prime} \cos k_{y}^{\prime}+\sin k_{x} \sin k_{y} \times\right.$ $\sin k_{x}^{\prime} \sin k_{y}^{\prime}$ ) and Hund's rule $-\frac{J_{H}}{N}$. The self-consistent equations are solved numerically. We find that in the region where $J_{H} \sim \operatorname{Max}\left(J_{1}, J_{2}\right), \Delta_{H}$ is extremely small. Hence, Hunds coupling has little effect on pairing symmetry. In the mixed $s_{x^{2} y^{2}}$ and $d_{x^{2}-y^{2}}$ phase, for $J_{1 ; 12} \lesssim J_{1}$ and $J_{2 ; 12} \lesssim J_{2}$, the orbital-crossing pairing order $\Delta^{\prime}$ is zero within computing error except for $d_{x y}$. We find that a coexisting small interorbital paring order with $d_{x y}$ symmetry, $\Delta^{\prime}(k)=$ $\Delta_{0}^{\prime} \sin \left(k_{x}\right) \sin \left(k_{y}\right)$. In Fig. 3, we plot the result for the intraorbital pairing order parameters $s_{x^{2} y^{2}}$ and $d_{x^{2}-y^{2}}$, and the interorbital pairing order parameter $d_{x y}$ as a function of $J=J_{1}=J_{2}=J_{1,12}=J_{2,12}$ when the chemical potential is $\mu=1.8$-corresponding to $18 \%$ electron doping. The result is a direct consequence of the $d_{x y}$ symmetry matching between the orbital-crossing pairing and the orbitalcrossing hopping term.

Discussion and Summary.-Although our prediction of the pairing symmetry is based on a two-orbital model, we believe it to be robust even if other orbitals are added. The pairing symmetry induced by the antiferromagnetic ex- change coupling is mainly determined by the structure of Fermi surfaces. As the Fermi surfaces in oxypnictides are located at $\Gamma$ and $M$ points, the $d_{x y}$ paring symmetry never wins over $s_{x^{2} y^{2}}$. As the Fe-based superconductors are in the regime $J_{2}>J_{1}[15,17]$, we predict an $s_{x^{2} y^{2}}=\cos \left(k_{x}\right) \times$ $\cos \left(k_{y}\right)$ order parameter in the superconducting state, which changes sign between the electron and hole Fermi surfaces. Moreover, we find that the interorbital pairing is small even if the orbital-crossing exchange is strong. Future research will focus on the effects of disorder on the superconducting state [26].

J.P.H. thanks S. Kivelson, E.W. Carlson, H. Yao, W. Tsai, C. Fang, D. Yao, and X. Tao for useful discussions. B. A. B. thanks P. W. Anderson for useful discussions. J. P. H. and K. J. S. were supported by the NSF under Grant No. PHY-0603759.

[1] Y. Kamihara, T. Watanabe, M. Hirano, and H. Hosono, J. Am. Chem. Soc. 130, 3296 (2008).

[2] Z.-A. Ren et al., Mater. Res. Innovations 12, 105 (2008).

[3] G. F. Chen et al., Phys. Rev. Lett. 101, 057007 (2008).

[4] X. H. Chen, et al., Nature (London) 453, 761 (2008).

[5] G. F. Chen et al., Phys. Rev. Lett. 100, 247002 (2008).

[6] H.-H. Wen, G. Mu, L. Fang, H. Yang, and X. Zhu, Europhys. Lett. 82, 17009 (2008).

[7] G. Mu et al., Chin. Phys. Lett. 25, 2221 (2008).

[8] L. Shan et al., Europhys. Lett. 83, (2008).

[9] R. H. Liu et al., Phys. Rev. Lett. 101, 087001 (2008).

[10] X. Dai et al., Phys. Rev. Lett. 101, 057008 (2008); G. Xu et al., Europhys. Lett. 82, 67002 (2008); K. Kuroki et al., Phys. Rev. Lett. 101, 087004 (2008); P. A. Lee and X.-G. Wen, arXiv:0804.1739; Q. Si and E. Abrahams, arXiv:0804.2480; Z.-J. Yao et al., arXiv:0804.4166; X.-L. Qi et al., arXiv:0804.4332.

[11] D. Singh and M.-H. Du, Phys. Rev. Lett. 100, 237003 (2008); G. Xu et al., Europhys. Lett. 82, 67002 (2008); I. I. Mazin et al., Phys. Rev. Lett. 101, 057003 (2008); K. Haule, J.H. Shim, and G. Kotliar, Phys. Rev. Lett. 100, 226402 (2008).

[12] C. de la Cruz et al., Nature (London) 453, 899 (2008).

[13] I. I. Mazin, D. J. Singh, M. D. Johannes, and M.-H. Du, Phys. Rev. Lett. 101, 057003 (2008).

[14] S. Raghu et al., Phys. Rev. B 77, 220503(R) (2008).

[15] F. Ma, Z. Lu, and T. Xiang, arXiv:0804.3370.

[16] T. Yildirim, Phys. Rev. Lett. 101, 057010 (2008).

[17] Q. Si and E. Abrahams, arXiv:0804.2480.

[18] C. Fang et al., Phys. Rev. B 77, 224509 (2008).

[19] C. Xu et al., Phys. Rev. B 78, 020501(R) (2008).

[20] Jian Li and Yupeng Wang, Chin. Phys. Lett. 25, 2232 (2008).

[21] S. Sachdev, Physica Amsterdam) 313A, 252 (2002).

[22] K. Seo, B. A. Bernevig, and J. Hu (unpublished).

[23] G. Kotliar, Phys. Rev. B 37, 3664 (1988).

[24] J. Dong et al., Europhys. Lett. 83, 27006 (2008).

[25] P. W. Anderson, Science 235, 1196 (1987).

[26] A. V. Balatsky, M. I. Salkola, and A. Rosengren, Phys. Rev. B 51, 15547 (1995). 